This is a copy of an article published in the Journal of Caffeine Research; copyright (C) 2012 Mary Ann Liebert, Inc.; Journal of Caffeine Research is available online at: http://online.liebertpub.com 


\title{
Caffeine Intake and the Risk of Chronic Obstructive Pulmonary Disease in Japanese Adults
}

\author{
Fumi Hirayama, ${ }^{1}$ Andy H. Lee, ${ }^{2}$ Keiji Yasukawa, ${ }^{1,3}$ Yukio Ishihara, ${ }^{1}$ and Masaki Shinjo ${ }^{4}$
}

Background: To investigate the association between caffeine intake and the risk of developing chronic obstructive pulmonary disease (COPD) among Japanese older adults, a case-control study was conducted in central Japan. Methods: A total of 277 patients with COPD aged 50 to 75 years were referred by respiratory physicians, while 340 controls were recruited from the community. All participants had their disease status confirmed by spirometry. Information on habitual caffeine intake, demographic, and lifestyle characteristics was obtained by face-to-face interview using a validated questionnaire.

Results: The COPD patients drank more coffee and had a higher mean caffeine intake (311.3, SD 176.2 mg/day) than the control group (278.4, SD $188.1 \mathrm{mg} /$ day), $p=0.03$. Relative to nondrinkers, the risk of COPD apparently increased for those drinking at least two cups of coffee daily (adjusted odds ratio 1.58, 95\% confidence interval [CI] 0.93 to 2.71). Similarly, total caffeine intake was associated with the prevalence of COPD—the adjusted odds ratio being 1.30 (95\% CI 1.09 to 2.12) for consuming over $312 \mathrm{mg} /$ day when compared to a low intake of less than $184 \mathrm{mg} /$ day.

Conclusions: The epidemiological evidence suggested a positive association between caffeine intake and the development of COPD in Japanese adults. Further study of the effect of caffeine on lung function and the risk of COPD should be undertaken.

\section{Introduction}

C HRONIC OBSTRUCTIVE PULMONARY DISEASE (COPD) is a leading cause of death and disability worldwide. ${ }^{1}$ It is characterized by a progressive irreversible air flow limitation with common symptoms that include chronic cough, sputum production, and dyspnoea on exertion. ${ }^{2}$ Although tobacco smoking is known to be the principal risk factor, $25 \%$ to $45 \%$ of patients with COPD have never smoked. ${ }^{2}$ Therefore, dietary habits, environmental exposure, and other host factors may contribute to the development of this disease. ${ }^{3,4}$ In Japan, the burden of COPD is expected to increase for the next decade due to continue cigarette smoking and the aging population. ${ }^{5}$ Research on the risk factors of COPD is thus important for the control and prevention of this disease.

The effects of caffeine and/or coffee consumption on the respiratory system have been inconsistent. A large populationbased cohort study in the USA found that nonsmokers who drank at least four cups of coffee per day have slightly better lung function than those who never or seldom drank coffee. ${ }^{6}$
It has also been suggested that caffeine can reduce respiratory muscle fatigue and asthma symptoms. ${ }^{7}$ While caffeine appears to have no deleterious effect on breathlessness, ${ }^{8}$ an experimental study showed that caffeine can stimulate the proliferation of human lung adenocarcinoma cells and small airway epithelial cells. ${ }^{9}$ Meta analyses further confirmed a significant positive association between coffee consumption and the lung cancer risk, ${ }^{10}$ with a linear dose-response relationship. ${ }^{11}$

Although caffeine ingestion has been suspected of contributing to the development of $\mathrm{COPD}^{12}$ the hypothesis has never been investigated in the literature. The objective of this research was to ascertain the strength of association between caffeine intake and the prevalence of COPD, by conducting a case-control study in Japan.

\section{Materials and Methods}

\section{Participants}

Three hundred COPD patients referred by respiratory physicians were recruited from the outpatient departments of six

\footnotetext{
${ }^{1}$ Medical Network Group, Innovation Center for Medical Redox Navigation, Kyushu University, Fukuoka, Japan

${ }^{2}$ School of Public Health, Curtin University, Perth, Australia.

${ }^{3}$ Department of Bio-function Science, Faculty of Pharmaceutical Sciences, Kyushu University, Fukuoka, Japan.

${ }^{4}$ Okinawa Prefectural College of Nursing, Naha, Okinawa, Japan.
} 
hospitals in central Japan (Aichi, Gifu, and Kyoto). Inclusion criteria for cases were as follows: (1) aged between 50 and 75 years; (2) had COPD as the primary functionally limiting illness that was diagnosed within the past 3 years. Patients were excluded if they had a recent stroke, dementia, or other health conditions that prohibited them from being interviewed. Twenty-three eligible patients were subsequently excluded due to missing or incomplete demographic and lifestyle details. The remaining 277 patients (244 men and 33 women) were available for analysis.

During the same period, 400 community-dwelling adults were recruited from the same catchment areas as the cases. These controls were approached and interviewed by the first author at shopping malls, community centers, or when they attended health checks at hospitals. They were selected to be frequency-matched to the prevalent cases by age (within 5 years). The same exclusion criteria as cases were applied, resulting in 340 eligible controls (272 men and 68 women). All participants underwent spirometric measurements of respiratory function to avoid misclassification of the casecontrol status. No significant differences were found between the included and excluded participants in terms of the available variables. Approval of the study protocol was obtained from the Human Research Ethics Committee of Curtin University. Permission to recruit patients and access to medical records were granted by the participating hospitals in Japan.

\section{Questionnaire and interview}

Information on habitual food and beverage consumption was obtained using a food frequency questionnaire developed by the Japan Epidemiological Association, ${ }^{13}$ with validity and reproducibility being established for Japanese adults. ${ }^{14}$ For each beverage type, such as coffee and green tea, participants were asked to report the average quantity consumed and frequency of intake in nine levels ranging from "almost never" to " 10 or more cups per day." Chocolate intake (g/day) was calculated by multiplying the frequency with the portion size per time. The reference recall for dietary variables was set at 5 years before interview because estimation beyond 5 years would be difficult.

The final part of the structured questionnaire collected information on demographic and lifestyle characteristics, including age, gender, weight, height, marital status, education level, smoking, alcohol drinking, as well as history of recent health conditions (hypertension, ischemic stroke, diabetes mellitus, depression, and cancer). For the COPD patients, each interview was conducted after making appointment with their respiratory physician. The purpose of the study was explained to each participant before obtaining their formal written consent. All interviews, averaging 40 minutes in duration, took place in the hospital outpatient departments for COPD patients and their place of recruitment for controls.

\section{Statistical analysis}

For each subject, total caffeine intake (mg/day) was obtained by adding the corresponding quantities from coffee, black tea, green tea, oolong tea, and chocolate consumption. One serving was defined as $140 \mathrm{~g}$ for coffee and black tea, $80 \mathrm{~g}$ for green tea, and $200 \mathrm{~g}$ for oolong tea. The caffeine contents of these items were taken from the Japanese standard tables of food composition, with $0.6 \mathrm{mg} / \mathrm{g}$ for coffee, $0.3 \mathrm{mg} / \mathrm{g}$ for black tea, $0.2 \mathrm{mg} / \mathrm{g}$ for green tea and oolong tea, and $0.125 \mathrm{mg} / \mathrm{g}$ for chocolate. ${ }^{15}$ A cup of coffee, for example, was thus assumed to contain $84 \mathrm{mg}$ of caffeine.

After comparing the case and control groups using chisquare and $t$ tests, unconditional logistic regression analyses were performed to determine the association between the prevalence of COPD and coffee drinking frequency, coffee consumption quantity, and total caffeine intake. The exposure variables were categorized into three levels of increasing consumption, with cut points of the categories corresponding to approximate tertiles for the distribution of controls. Each regression model accounted for the effects of age, gender, body-mass index (BMI), smoking status (never smoker, former smoker, current smoker), alcohol drinking (nondrinker, drinker), education (high school or below, college or university), and presence of a health condition. These variables were considered potential confounders from the literature. All statistical analyses were undertaken using the SPSS package version 20.0.

\section{Results}

Table 1 presents the demographic and lifestyle characteristics of the participants. The majority of them were male $(83 \%)$ and married $(84 \%)$, with mean age about 66 years. For the case group, 220 patients $(80 \%)$ had their COPD diagnosed within the past 2 years. The COPD patients were on average 1 year older and had lower BMI (5 years ago) than the control subjects. They also appeared to be less educated and non-

Table 1. Characteristics of Participants by Case-Control Status

\begin{tabular}{|c|c|c|c|}
\hline & COPD & Control & $\mathrm{p}$ \\
\hline$N$ & 277 & 340 & \\
\hline Age: mean (SD), years & $66.47(6.75)$ & $65.35(5.49)$ & 0.028 \\
\hline $\begin{array}{l}\text { Body-mass index: } \\
\text { mean (SD), } \mathrm{kg} / \mathrm{m}^{2}\end{array}$ & $21.94(3.09)$ & $23.54(2.94)$ & $<0.001$ \\
\hline Gender & & & 0.007 \\
\hline Male & $244(88.1 \%)$ & $272(80.0 \%)$ & \\
\hline Female & $33(11.9 \%)$ & $68(20.0 \%)$ & \\
\hline Education $^{\mathrm{a}}$ & & & $<0.001$ \\
\hline High school or below & $221(80.4 \%)$ & $213(63.6 \%)$ & \\
\hline College or university & $54(19.6 \%)$ & $122(36.4 \%)$ & \\
\hline Marital status ${ }^{\mathrm{a}}$ & & & 0.881 \\
\hline Single or divorced & $43(15.6 \%)$ & $54(16.0 \%)$ & \\
\hline Married & $233(84.4 \%)$ & $283(84.0 \%)$ & \\
\hline Health condition ${ }^{a, b}$ & & & 0.988 \\
\hline Absence & $134(48.7 \%)$ & $164(48.7 \%)$ & \\
\hline Presence & $141(51.3 \%)$ & $173(51.3 \%)$ & \\
\hline Smoking status ${ }^{\mathrm{a}}$ & & & $<0.001$ \\
\hline Current smoker & $62(22.5 \%)$ & $65(19.2 \%)$ & \\
\hline Former smoker & $207(75.3 \%)$ & $136(40.1 \%)$ & \\
\hline Never smoker & $6(2.2 \%)$ & $138(40.7 \%)$ & \\
\hline Alcohol drinking ${ }^{a, c}$ & & & 0.026 \\
\hline Nondrinker & $119(43.0 \%)$ & $116(34.2 \%)$ & \\
\hline Drinker & $158(57.0 \%)$ & $223(65.8 \%)$ & \\
\hline
\end{tabular}

${ }^{\mathrm{a}}$ Missing data present.

${ }^{\mathrm{b}}$ Recent history of hypertension, ischemic stroke, diabetes mellitus, depression, or cancer.

${ }^{\mathrm{c}}$ Nondrinker: less than once per month; drinker: at least once per month.

COPD, chronic obstructive pulmonary disease. 
TAble 2. Comparison of Caffeine Intake from Various Sources Between Case and Control Groups

\begin{tabular}{lccc}
\hline & COPD $(\mathrm{n}=277)$ & Control $(\mathrm{n}=340)$ & \\
Caffeine sources & Mean $(S D), m g /$ day & Mean $(S D)$, mg/day & $\mathrm{p}$ \\
\hline Coffee & $148.22(135.23)$ & $111.89(133.35)$ & 0.001 \\
Black tea & $2.89(10.74)$ & $3.84(11.65)$ & 0.299 \\
Green tea & $136.12(111.05)$ & $139.93(119.52)$ & 0.690 \\
Oolong tea & $24.81(66.51)$ & $23.79(67.36)$ & 0.851 \\
Chocolate & $0.24(0.60)$ & $0.26(0.60)$ & 0.595 \\
Total intake & $311.33(176.19)$ & $278.39(188.06)$ & 0.030 \\
\hline
\end{tabular}

COPD, chronic obstructive pulmonary disease.

alcohol drinkers relative to their counterparts without the disease. A significantly higher proportion of former smokers was observed among COPD patients than among controls.

Table 2 compares the caffeine intake between case and control subjects. Mean total caffeine intake was significantly higher among the COPD patients (311.3, SD $176.2 \mathrm{mg} /$ day) than among the controls (278.4, SD $188.1 \mathrm{mg} /$ day), which might be attributed to the difference in coffee consumption $(p=0.001$ ) between the two groups (mean 247, SD $225 \mathrm{~mL} /$ day versus mean 186, SD $222 \mathrm{~mL}$ /day, respectively).

Table 3 presents the logistic regression results. Apparent increase in COPD risk was observed for frequent coffee drinking of at least two cups or over $140 \mathrm{~mL}$ daily, when compared to nondrinkers or those with low consumption less than $30 \mathrm{~mL} /$ day. Similarly, total caffeine intake was positively associated with the COPD prevalence; the linear dose-response relationship remained significant $(p=0.03)$ after accounting for the effects of confounding variables.

\section{Discussion}

The present study provides the first report on the association between caffeine intake and the risk of COPD. The Japanese Food Safety Commission has recommended an upper limit of caffeine intake at $400 \mathrm{mg} /$ day for healthy adults. ${ }^{16}$ It should be remarked that $28 \%$ of our participants $(34 \%$ of cases and $23 \%$ of controls) had exceeded this limit.

Our results showed that both coffee consumption and caffeine intake are positively associated with the prevalence of
COPD. It has been hypothesized that caffeine contributes to the development of chronic airflow obstruction and plays a role in the genesis of bronchial cancer, despite its beneficial effect on bronchospasm. ${ }^{12}$ Emerging epidemiological evidence has also suggested that high levels of coffee consumption may increase the risk of lung cancer, ${ }^{10,11,17}$ possibly by stimulating the proliferation of lung adenocarcinoma cells and small airway epithelial cells. ${ }^{9}$ Therefore, the carcinogenic effect of caffeine may outweigh the potentially protective effect of phytochemicals present in coffee. ${ }^{17}$ Since caffeine possesses a variety of pharmacological effects, more research is needed to understand the biological mechanism underlying the relationship between caffeine and the development of COPD.

The effects of confounding factors have been accounted for in the logistic regression models. It should be remarked that classifying individuals as either "drinker" or "nondrinker" has provided little information on their actual alcohol use. Moreover, the relationship between caffeine and alcohol is complex, and can be mediated by smoking status. ${ }^{18,19}$ For our participants, caffeine intake and alcohol drinking were moderately associated $(p=0.04)$ with higher caffeine intake by nondrinkers (312.9, SD $182.3 \mathrm{mg} /$ day) than drinkers (281.0, SD $183.2 \mathrm{mg} /$ day), but the difference between nondrinkers (327.0, SD $179.5 \mathrm{mg} /$ day) and drinkers (279.3, SD $178.0 \mathrm{mg} /$ day) was more prominent $(p=0.02)$ among former smokers.

In this study, the sample size of 617 subjects allowed sufficient power for the multivariable analysis. A validated and reliable food frequency questionnaire for Japanese adults was administered to obtain information on habitual food and beverage consumption, without performing direct measurements of plasma or urinary caffeine level. Another strength of the study was that spirometric measurements were taken to ensure correct classification of the case-control status. All interviews and assessments were conducted by the same investigator (first author) to eliminate inter-interviewer bias.

Several limitations should be taken into consideration. The retrospective cross-sectional study design posed a major limitation so that any cause-effect relationship could not be established. The adverse effect of caffeine was not established for COPD and all participants were blinded to the study hypothesis, so that information bias was unlikely. Although

Table 3. Coffee Consumption, Caffeine Intake, and Risk of COPD for Japanese Adults

\begin{tabular}{|c|c|c|c|c|c|}
\hline Variable & COPD & Control & Crude OR (95\% CI) & Adjusted $O R^{\mathrm{a}}(95 \% \mathrm{CI})$ & $\mathrm{p}^{\mathrm{a}}$ \\
\hline Coffee drinking frequency & & & & & 0.08 \\
\hline Never & $51(18.4 \%)$ & $87(26.0 \%)$ & 1 & 1 & \\
\hline $1-2$ times per week to 1 cup daily & $103(37.2 \%)$ & $142(42.4 \%)$ & $1.24(0.81,1.90)$ & $1.19(0.70,2.03)$ & \\
\hline 2 or more cups daily & $123(44.4 \%)$ & $106(31.6 \%)$ & $1.98(1.29,3.05)$ & $1.58(0.93,2.71)$ & \\
\hline Coffee consumption quantity (mL/day) & & & & & 0.04 \\
\hline$<30$ & $68(24.5 \%)$ & $114(34.0 \%)$ & 1 & 1 & \\
\hline 30 to 140 & $86(31.0 \%)$ & $115(34.3 \%)$ & $1.25(0.83,1.89)$ & $1.37(0.82,2.28)$ & \\
\hline$>140$ & $123(44.4 \%)$ & $106(31.6 \%)$ & $1.95(1.31,2.89)$ & $1.68(1.02,2.75)$ & \\
\hline \multicolumn{6}{|l|}{ Total caffeine intake (mg/day) } \\
\hline$<184$ & $77(28.5 \%)$ & $112(33.9 \%)$ & 1 & 1 & 0.03 \\
\hline 184 to 312 & $62(23.0 \%)$ & $106(32.1 \%)$ & $0.85(0.56,1.30)$ & $0.66(0.39,1.13)$ & \\
\hline$>312$ & $131(48.5 \%)$ & $112(33.9 \%)$ & $1.70(1.16,2.50)$ & $1.30(1.09,2.12)$ & \\
\hline
\end{tabular}

adjusted for age, gender, body-mass index, health condition (absence, presence), education (high school or below, college or university), smoking status (current smoker, former smoker, never smoker), and alcohol drinking (nondrinker, drinker).

$\mathrm{CI}$, confidence interval; OR, odds ratio. 
recall error could not be eliminated, face-to-face interviews were conducted to help the recall of food and beverage consumption and to avoid misinterpretation of the questions. The community-dwelling controls were recruited during the same period and from the same catchment area as the cases. They should be representative of the elderly Japanese population. However, the inherent selection bias still existed because of their voluntary participation in the study. Reverse causation was a possibility even though the reference time point for dietary intake was set at 5 years before interview, and no participant admitted any change in dietary habits within the past 5 years. For this sample of participants, significant differences in demographic variables were observed between case and control groups, but over-matching of demographic characteristics could lead to recruitment difficulty if many potential controls were deemed ineligible. Finally, we noted the small effect sizes $(<0.3)$ despite the statistically significant differences in mean coffee consumption and mean caffeine intake between the two groups.

\section{Conclusions}

This case-control study suggested that Japanese adults with a high caffeine intake are susceptible to an increased risk of COPD. Further replications in other populations and large prospective cohort studies are recommended to confirm the effect of caffeine intake on the development of COPD.

\section{Author Disclosure Statement}

No competing financial interests exist.

\section{References}

1. World Health Organization. Chronic Obstructive Pulmonary Disease (COPD). 2010; Available from: www.who.int/en

2. Global Initiative for Chronic Obstructive Lung Disease. Global Strategy for the Diagnosis, Management, and Prevention of Chronic Obstructive Pulmonary Disease. 2011; Available from: www.goldcopd.org/uploads/users/files/GOLD_Report_ 2011_Feb21.pdf

3. Hirayama F, Lee AH, Binns CW. Dietary factors for chronic obstructive pulmonary disease: epidemiological evidence. Expert Rev Respir Med. 2008;2:645-653.

4. Hirayama F, Lee AH, Hiramatsu N. Dietary nutrients in relation to chronic obstructive pulmonary disease: Emerging epidemiological evidence. Curr Respir Med Rev. 2010;6:124-132.

5. Teramoto S, Yamamoto H, Yamaguchi Y, Matsuse T, Ouchi Y. Global burden of COPD in Japan and Asia. Lancet. 2003;362:1764-1765.

6. Nettleton JA, Follis JL, Schabath MB. Coffee intake, smoking, and pulmonary function in the Atherosclerosis Risk in Communities Study. Am J Epidemiol. 2009;169:1445-1453.

7. Welsh EJ, Bara A, Barley E, Cates CJ. Caffeine for asthma. Cochrane Database Syst Rev. 2010:CD001112.
8. Woodcock AA, Gross ER, Gellert A, Shah S, Johnson M, Geddes DM. Effects of dihydrocodeine, alcohol, and caffeine on breathlessness and exercise tolerance in patients with chronic obstructive lung disease and normal blood gases. N Engl J Med. 1981;305:1611-1616.

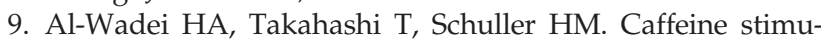
lates the proliferation of human lung adenocarcinoma cells and small airway epithelial cells via activation of PKA, CREB and ERK1/2. Oncol Rep. 2006;15:431-435.

10. Tang N, Wu Y, Ma J, Wang B, Yu R. Coffee consumption and risk of lung cancer: a meta-analysis. Lung Cancer. 2010; 67:17-22.

11. Wang Y, Yu X, Wu Y, Zhang D. Coffee and tea consumption and risk of lung cancer: a dose-response analysis of observational studies. Lung Cancer. 2012;78:169-170.

12. Martinet Y, Debry G. Effects of coffee on the respiratory system. Rev Mal Respir. 1992;9:587-592.

13. Ishihara J, Sobue T, Yamamoto S, Sasaki S, Tsugane S. Demographics, lifestyles, health characteristics, and dietary intake among dietary supplement users in Japan. Int J Epidemiol. 2003;32:546-553.

14. Ishihara J, Sobue T, Yamamoto S, Yoshimi I, Sasaki S, Kobayashi $\mathrm{M}$, et al. Validity and reproducibility of a selfadministered food frequency questionnaire in the JPHC study cohort II: study design, participant profile and results in comparison with cohort I. J Epidemiol. 2003;13:S134-S147.

15. Ministry of Education, Culture, Sports Science and TechnologyJapan. Standard Tables of Food Composition in Japan, fifth revised and enlarged edition. 2005; Available from: www.mext.go.jp/ b_menu/shingi/gijyutu/gijyutu3/toushin/05031802.htm

16. Food Safety Commission. Caffeine Content in Foods. 2011; Available from: www.fsc.go.jp/sonota/factsheets/ caffeine.pdf

17. Baker JA, McCann SE, Reid ME, Nowell S, Beehler GP, Moysich KB. Associations between black tea and coffee consumption and risk of lung cancer among current and former smokers. Nutr Cancer. 2005;52:15-21.

18. Istvan J, Matarazzo JD. Tobacco, alcohol and caffeine use: a review of their interrelationships. Psychol Bull. 1984;95: 301-326.

19. Gurpegui M, Aguilar MC, Martinez-Ortega JM, Jurado D, Diaz FJ, Quintana HM, et al. Fewer but heavier caffeine consumers in schizophrenia: a case-control study. Schizophr Res. 2006;86:276-283.

Address correspondence to: Andy H. Lee, PhD School of Public Health Curtin University GPO Box U 1987

Perth, WA 6845

Australia

E-mail: andy.lee@curtin.edu.au 